\title{
Dynamics of optically injected currents in carbon nanotubes
}

\author{
L. L. Bonilla, ${ }^{1}$ M. Alvaro, ${ }^{1}$ M. Carretero, ${ }^{1}$ and E. Ya. Sherman ${ }^{2,3}$ \\ ${ }^{1}$ Gregorio Millán Institute for Fluid Dynamics, Nanoscience and Industrial Mathematics, \\ Universidad Carlos III de Madrid, Avenida de la Universidad 30, 28911 Leganés, Spain \\ ${ }^{2}$ Department of Physical Chemistry, The University of the Basque Country, 48080 Bilbao, Spain \\ ${ }^{3}$ IKERBASQUE Basque Foundation for Science, Bilbao, Spain
}

\begin{abstract}
We consider theoretically the dynamics of electric currents optically injected in carbon nanotubes. Although the plasma oscillations are not seen in these systems, the main effect on the carrier's motion is due to strongly nonuniform space-charge Coulomb forces produced by time-dependent separation of injected electron and hole densities. We calculate evolution of the dipole moment characterizing the time- and coordinate-dependent charge density distributions and analyze different regimes of the dynamics. The developed time-dependent dipole moment leads to a dipole radiation in the $\mathrm{THz}$ frequency range for typical parameters of injected currents.
\end{abstract}

PACS numbers: 73.63.Fg, 78.67.Ch, 42.65.Re

\section{INTRODUCTION}

Optical manipulation of carriers in bulk solids and artificial structures is an interesting problem for fundamental and applied physics. By interference of singleand two-photon optical transitions induced by highly coherent laser beams, which can be controlled by the beams' phases, one can inject optically currents in semiconductors ${ }^{112}$ and semiconductor nanostructures ${ }^{3 / 4}$. Recently, current injection using the same principle of interference was reported for a different class of systems such as graphene ${ }^{5}$ and carbon nanotubes ${ }^{6}$. The understanding of the following dynamics of the injected currents can provide a valuable information both about the injection process and interactions in the system.

For two-dimensional semiconductor quantum wells 7 it was shown that the space-charge effects due to nonuniform charge density play the crucial role in the electron motion while relatively heavy holes can be taken at rest. If the space-charge effects dominate in the charge dynamics, the timescale of the evolution is given by the characteristic expected plasma frequency corresponding to the injected charge density and the laser spot size. However, the plasma oscillations should not be seen there since a highly nonuniform charge density is formed on the timescale of the order of the expected inverse plasma frequency.

In this respect, carbon nanotubes are strongly different from conventional semiconductors. Semiconducting nanotubes, where carriers have finite effective masses, were intensively investigated by optical techniques. Near the absorption threshold they demonstrate excitonic effects in the optical absorption spectra ${ }^{8}$ and in the subsequent dynamics ${ }^{9}$. Here electrons and holes give the same contribution to the optical properties. Another type of nanotubes is metallic systems, where the dispersion relation of carriers is linear in the momentum, making the plasma frequency a poorly defined quantity. As a result, even when the carrier momentum changes due to the relaxation and external forces, the velocity, and there- fore the current, can remain constant. To change the carrier velocity the momentum has to change sign. In general, for these "relativistic" spectra, even relatively strong Coulomb forces do not lead to formation of excitons (for an exception, see Ref.[10]). This new type of dynamics, which will be of our interest here, can experimentally be seen in bunches of nanotubes containing metallic and semiconducting species. As we are interested in the optical response in the relatively low frequency infrared domain, semiconducting nanotubes will not contribute to the properties of our interest. Moreover, metallic nanotubes can be separated from the semiconducting ones ${ }^{11}$, to provide a system for experimental study of the effects considered here.

A microscopic theory of current injection in semiconducting nanotubes has been developed ${ }^{12}$ by using the analysis of the transition matrix elements on the atomic scale. However, the stage of the subsequent dynamics with a strongly nonuniform density has not yet been studied and understood. Here we study this process. The time-dependent injected current is accompanied by emission of radiation in the $\mathrm{THz}$ frequency domain. As we will show, the spectrum of this radiation provides information about the dynamics and properties of the system.

\section{MODEL DYNAMICS EQUATIONS}

We begin with model equations for a single wall carbon nanotube characterized by dispersion relation (see Fig. 1):

$$
\varepsilon(k)=\hbar|k| v_{0}, \quad \varepsilon(k)=-\hbar|k| v_{0},
$$

for electrons and holes, respectively, and velocities $v(k)=$ $\pm \operatorname{sign}(k) v_{0}$ where $v_{0}=10^{8} \mathrm{~cm} / \mathrm{s}$.

Optical injection produces electron and hole densities $n^{ \pm}, p^{ \pm}$respectively in the coordinate $(x)$ and momentum $(k)$ - space wit corresponding velocities $v$ :

$$
\begin{array}{ll}
n^{+}=n^{+}(x, k, t) ; & p^{-}=p^{-}(x, k, t) ; \quad v=v_{0} \\
n^{-}=n^{-}(x, k, t) ; & p^{+}=p^{+}(x, k, t) ; \quad v=-v_{0}
\end{array}
$$




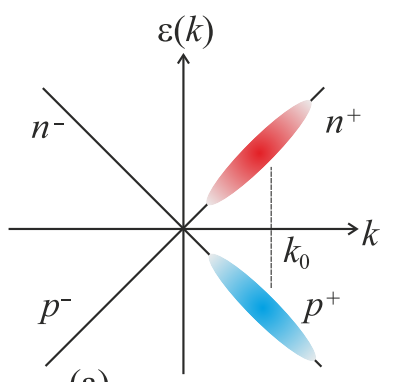

(a)

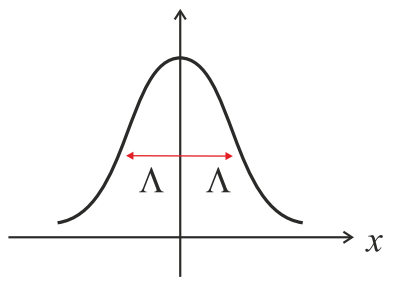

(b)
Figure 1: (a) Dispersion relation and density distribution in the momentum space. The peak in the optical radiation intensity corresponds to transitions at frequency $2 v_{0} k_{0}$. (b) Electron/hole density induced by the laser spot vs $x$, where $2 \Lambda$ is the total spot width.

The local densities are defined as:

$$
\begin{array}{cc}
\bar{n}^{ \pm}=\bar{n}^{ \pm}(x, t)=\int_{-\infty}^{+\infty} n^{ \pm}(x, k, t) d k, & \bar{n}=\bar{n}^{+}+\bar{n}^{-} ;(4) \\
\bar{p}^{ \pm}=\bar{p}^{ \pm}(x, t)=\int_{-\infty}^{+\infty} p^{ \pm}(x, k, t) d k, & \bar{p}=\bar{p}^{+}+\bar{p}^{-} .(5)
\end{array}
$$

In what follows we omit the explicit $(x, t, k)$-dependence for brevity. The Boltzmann equations for the distribution functions have the form

$$
\begin{gathered}
\frac{\partial n^{ \pm}}{\partial t}+v(k) \frac{\partial n^{ \pm}}{\partial x}+\frac{e E}{\hbar} \frac{\partial n^{ \pm}}{\partial k}=-\frac{n^{ \pm}-n_{e q}^{ \pm}}{\tau_{n}} \\
\frac{\partial p^{ \pm}}{\partial t}-v(k) \frac{\partial p^{ \pm}}{\partial x}-\frac{e E}{\hbar} \frac{\partial p^{ \pm}}{\partial k}=-\frac{p^{ \pm}-p_{e q}^{ \pm}}{\tau_{p}}
\end{gathered}
$$

where $e<0$ is the electron charge and $E \equiv E(x, t)$ is the coordinate- and time-dependent electric field produced by space-charge effects, that is by nonuniform charge density. The local Fermi-Dirac equilibrium densities for electrons in Eq. (6) and Eq. (7) are defined as

$$
n_{e q}^{ \pm}=2 \times \frac{1}{\exp \left[\left(\varepsilon(k)-\mu_{n}^{ \pm}\right) / T\right]+1},
$$

where the factor 2 is due to spin degeneracy and $T$ is the temperature measured in units of energy. In a similar way we define the equilibrium distributions of holes. The coordinate- and time-dependent chemical potentials $\mu_{n}^{ \pm}$ and $\mu_{p}^{ \pm}$guarantee the balance of the densities in the form $\bar{n}_{e q}^{ \pm}=\bar{n}^{ \pm}, \bar{p}_{e q}^{ \pm}=\bar{p}^{ \pm}$. For example, condition

$$
\int_{0}^{\infty} n_{e q}^{+} d k=\bar{n}^{+}
$$

yields

$$
\mu_{n}^{+}=T \ln \left[\exp \left(\hbar v_{0} \bar{n}^{+} / 2 T\right)-1\right]
$$

The electric field in Eqs. (6) and (7) can be expressed in terms of the integral of the charge density $\bar{p}(x-s, t)-$ $\bar{n}(x-s, t)$ as:

$$
E(x, t)=\int_{-\infty}^{\infty}[\bar{p}(x-s, t)-\bar{n}(x-s, t)] \mathcal{K}(s) d s,
$$

where $\mathcal{K}(s)$ is the Coulomb kernel for the nanotube. The expression for $\mathcal{K}(s)$ is presented in the Appendix. As we will see, the important and unusual feature of Eq. (11) is that in the limit of a small-radius nanotube the field $E(x, t)$ is proportional to the local derivative of the density: $\partial(\bar{p}(x, t)-\bar{n}(x, t)) / \partial x$.

The initial distributions of electrons and holes are optically produced as:

$$
n(x, k, 0)=p(x, k, 0)=\frac{N}{\pi \Lambda K} \exp \left[-\frac{x^{2}}{\Lambda^{2}}-\frac{\left(k-k_{0}\right)^{2}}{K^{2}}\right],
$$

where $k_{0}$ is the injection point in the momentum space, $2 \Lambda$ is the characteristic laser spot size, and $N$ is the total number of injected electron/holes. We use $k_{0}=K=200$ $\mu \mathrm{m}^{-1}$ (see Fig. 1(a)). This is reasonable since the wave vector is limited by the requirement that the carrier energy should not exceed that of the optical phonon, for otherwise a fast momentum and energy relaxation occur. A typical optical phonon energy is $\hbar \Omega_{\mathrm{ph}}=0.18 \mathrm{eV}$, 14 that gives the estimate $k_{0}<\Omega_{\mathrm{ph}} / v_{0}=275 \mu \mathrm{m}^{-1}$. Integrating Eq. 12 over $k$, we obtain (see Fig. 1(b)):

$$
\bar{n}(x, 0)=\bar{p}(x, 0)=\frac{N}{\sqrt{\pi} \Lambda} \exp \left(-x^{2} / \Lambda^{2}\right) .
$$

We define the one-dimensional (1D) electron/hole density

$$
N_{1 D}=\frac{N}{2 \Lambda},
$$

and Eq. 13 becomes:

$$
\bar{n}(x, 0)=\bar{p}(x, 0)=\frac{2 N_{1 D}}{\sqrt{\pi}} \exp \left(-x^{2} / \Lambda^{2}\right) .
$$

Integrating Eqs. (6) and (7) over $k$, we get the charge continuity equation:

$$
\begin{aligned}
& \frac{\partial}{\partial t}\left[\left(\bar{p}^{+}+\bar{p}^{-}\right)-\left(\bar{n}^{+}+\bar{n}^{-}\right)\right] \\
& \quad+v_{0} \frac{\partial}{\partial x}\left[\left(\bar{p}^{-}-\bar{p}^{+}\right)+\left(\bar{n}^{-}-\bar{n}^{+}\right)\right]=0,
\end{aligned}
$$

from which we define the local current:

$$
I=I(x, t)=-e v_{0}\left[\left(\bar{p}^{-}-\bar{p}^{+}\right)+\left(\bar{n}^{-}-\bar{n}^{+}\right)\right] .
$$

\section{NUMERICAL SOLUTIONS}

Before solving numerically the model equations, we introduce parameters describing the electron-electron interaction and the injection process. First we introduce 
a parameter characterizing the strength of the Coulomb forces. For this purpose we use the following scaling argument. The Coulomb force acting at a carrier, $F \sim$ $N e^{2} / \sqrt{\epsilon_{\perp} \epsilon_{\|}} \Lambda^{2}$ where $\epsilon_{\perp}$ and $\epsilon_{\|}$is the nanotube transversal and longitudinal permittivity, respectively (see Appendix for details). In the absence of the plasma frequency and on the relevant time scale $t_{\Lambda} \sim \Lambda / v_{F}$, this force produces a change in the momentum comparable to $\hbar K$ if $F t_{\Lambda} \sim \hbar K$. This estimate yields the corresponding critical number of injected carriers per nanotube $N_{c} \equiv \sqrt{\epsilon_{\perp} \epsilon_{\|}} \hbar v_{0} K \Lambda / e^{2} \approx 1600$ (for $K=200$ $\mu \mathrm{m}^{-1}, \epsilon_{\perp}=10$ and $\epsilon_{\|}=30$, in agreement with the experiment 13 ), and the interaction effects are described by a dimensionless parameter $N / N_{c}$. If $N / N_{c} \ll 1$, then $\bar{n}^{ \pm}$ and $\bar{p}^{ \pm}$are conserved and the initial density distributions move and separate being only weakly deformed. Otherwise, the effect of Coulomb forces is strong. In this case, if $E<0(E>0)$ then $\bar{n}^{+}$and $\bar{p}^{-}$increase (decrease), and $\bar{p}^{+}$and $\bar{n}^{-}$decrease (increase). Since initially carriers are injected with positive momentum $k_{0}>0$ most of the electrons/holes have $k>0$. Thus $n(x, 0, t)$ increases and $p(x, 0, t)$ decreases when $E>0$, and therefore the effect of the electric field is larger on the electrons than on the holes. Conversely, when $E<0$ the effect of the electric field is larger on the holes than on the electrons. However, when the number of electrons/holes having $k<0$ is larger, the sign of the electric field has the opposite effect for those electrons/holes.

An important limit on the number of injected particles is posed by the Pauli blocking condition, where the injection stops since all available electron/hole states became occupied by the previously excited carriers. The condition that the transition does not experience Pauli blocking limits the number of injected particles to the available phase volume $2 \Lambda \Delta K$, where 2 is the spin factor. Therefore, the maximum ratio $N / N_{c}$ should be considerably less than $(\Delta K / K) \alpha\left(c / v_{F}\right)\left(2 / \sqrt{\epsilon_{\perp} \epsilon_{\|}}\right)$, where $\alpha \equiv e^{2} / \hbar c=1 / 137$ is the fine structure constant. Provided $\Delta K \sim K=275 \mu \mathrm{m}^{-1}$, we obtain that $N$ should be less than 400 restricting $N / N_{c}$ to values considerably less than 0.3 .

We consider current injection by tightly focused beams with $\Lambda=1 \mu \mathrm{m}$, and nanotube of the radius $a=1.25$ and solve numerically the equations (6) to (11) in the following two cases (see Fig. 2-Fig. 5): (a) $N_{1 D} \approx 1.25 \times$ $10^{5} \mathrm{~cm}^{-1}$, injected $N=25, N / N_{c} \approx 0.016$, and $I_{\max } \approx 4$ $\mu \mathrm{A}$ and (b) $N_{1 D} \approx 1.25 \times 10^{6} \mathrm{~cm}^{-1}, N=250, N / N_{c} \approx$ 0.16 , and $I_{\max } \approx 40 \mu \mathrm{A}$. In both cases we assume carriers scattering times $\tau_{n}=\tau_{p}=2$ ps at $300 \mathrm{~K}$, as suggested by the estimates $15 \mid 16$.

In case (a), there are few carriers and electrons and holes go their separate ways without much interaction, as shown in Fig. 2. The effect of the non-equilibrium electric field is much greater in case (b), when there are ten times more carriers. Figure 3 shows that the interaction between carriers builds up a peak in the hole density. The extrema of the electric field are reached at the inflection points of the charge density $\bar{p}(x, t)-\bar{n}(x, t)$ in
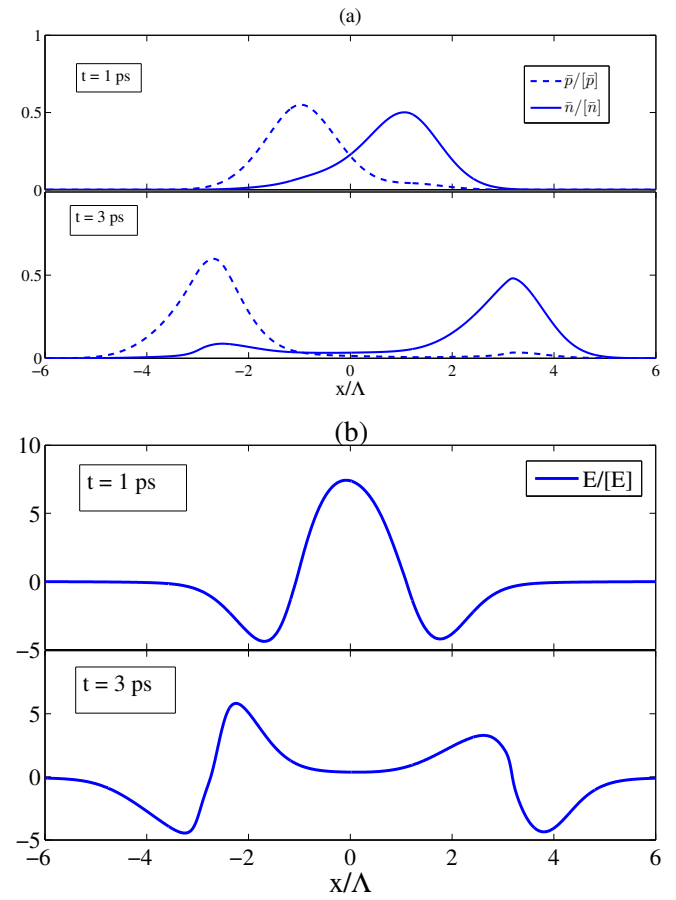

Figure 2: Profiles of (a) density of electrons/holes, and (b) electric field at different snapshots for case a): $\Lambda=1 \mu \mathrm{m}$, $N_{1 D} \approx 12.5 \mu \mathrm{m}^{-1}, \tau_{p}=\tau_{n}=2 \mathrm{ps}, N=25$ electrons/holes. Here $[\bar{p}]=[\bar{n}]=N / \Lambda=2.5 \times 10^{5} \mathrm{~cm}^{-1}$, and the unit of electric field $[E] \equiv|e| N / \epsilon_{\perp} \Lambda^{2}=36 \mathrm{~V} / \mathrm{cm}$.

agreement with the approximate formulas presented in the Appendix. The displacement and separation of electron and hole peaks is of the order of the initial width $\Lambda$, that is much larger than can observed in semiconductor quantum wells 37 . The reduction of separation by space-charge effects due to the finite mass of the carriers, similar to that in the quantum wells, can be expected in semiconductor nanotubes as well. As one can see in the Figures for carrier densities and electric fields, the scattering sharpens the peaks in the carrier densities and the electric field and depresses the smooth regions thereof but does not change this qualitative picture. The reason is that the scattering tries to keep the carrier densities close their local equilibrium values (Fermi functions) which have large gradients near $k=0$. This enhances the effect of the electric field on the densities and sharpens their peaks.

Figure 3(b) shows the development of electric fields and, thus, details the way the carriers peaks are built. Since we inject current with positive momentum, the larger peaks of electron and hole densities correspond to $k>0$. However, the hole population with $k<0$ splits in two parts and one part moves together with the hole population with $k>0$ which helps building up the hole population at the peak that moves to the left. Meanwhile, the electron population with $k>0$ also splits in two parts and the one that moves to the left helps rein- 


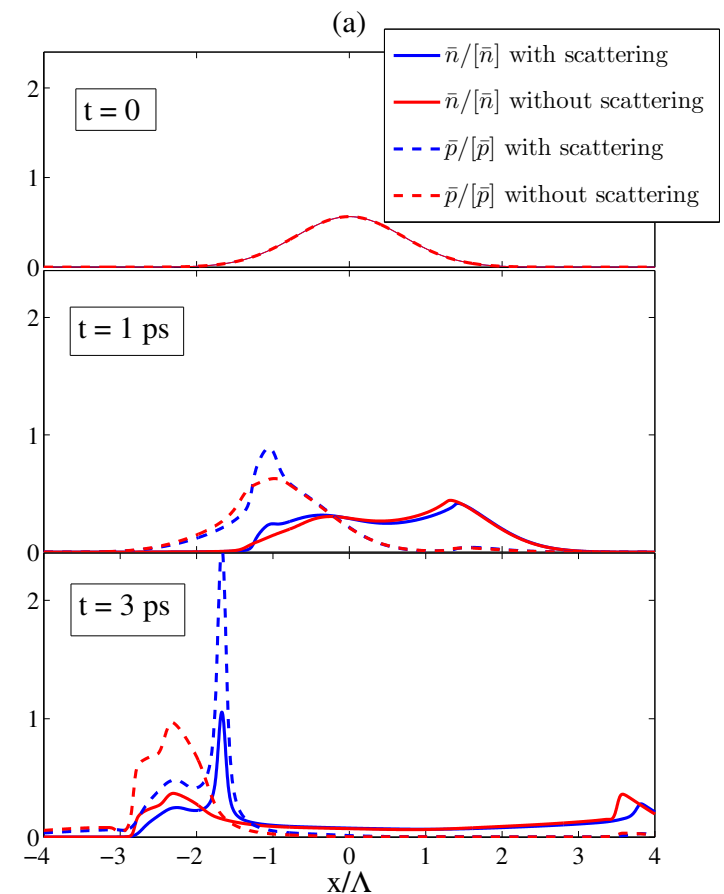

(b)

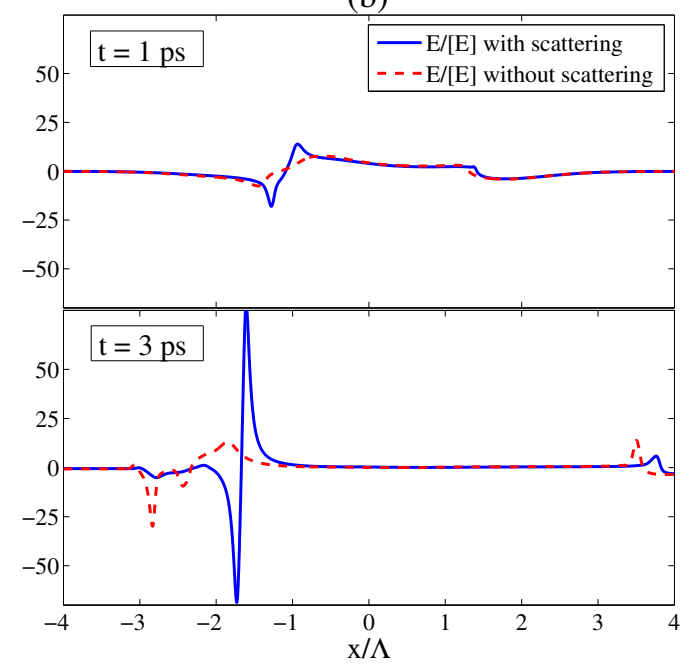

Figure 3: (a) Profiles of the electron/hole densities, and (b) electric field profile, at different snapshots, for $N=250$ electrons/holes. Here $[\bar{p}]=[\bar{n}]=N / \Lambda=2.5 \times 10^{6} \mathrm{~cm}^{-1}$, and the unit of electric field $[E] \equiv|e| N / \epsilon_{\perp} \Lambda^{2}=360 \mathrm{~V} / \mathrm{cm}$.

forcing the electron population with $k<0$. The location of the electron peak that moves to the left is quite close to that of the left-moving hole peak. Then electrons and holes interact so that their left-moving peaks slow down almost to a halt at the same location.

This picture is confirmed in Fig. 4 that shows snapshots of the overall hole density $p^{+}(x, k, t)+p^{-}(x, k, t)$ for $N=250$ electron/holes. Note that the Coulomb forces stop the motion of carriers to the left and build up hole and electron peaks at $x \approx 2.3 \Lambda$. Similarly, increasing the number of carriers narrows the peaks of their spatial (a)

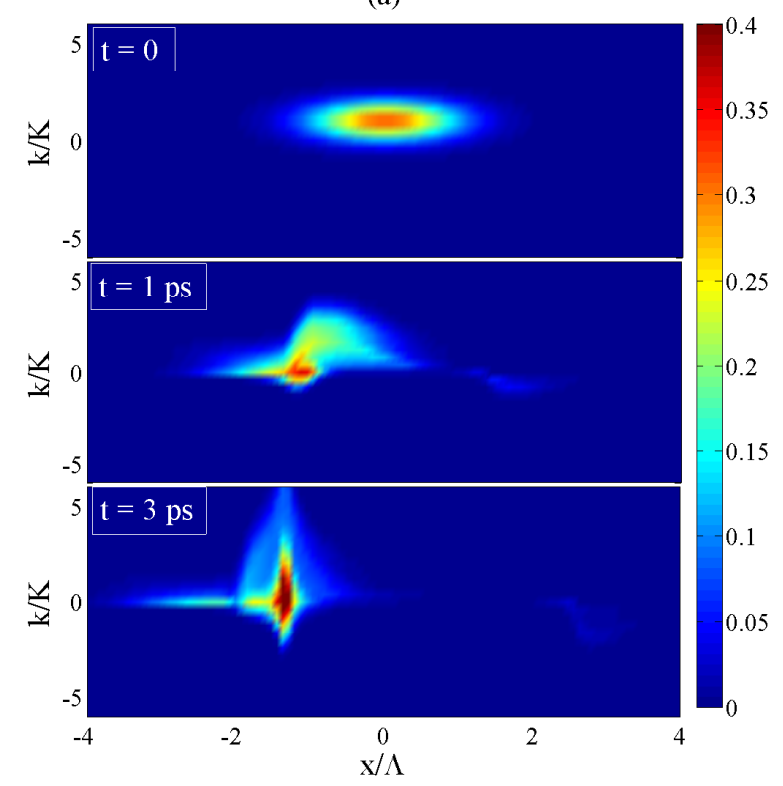

(b)

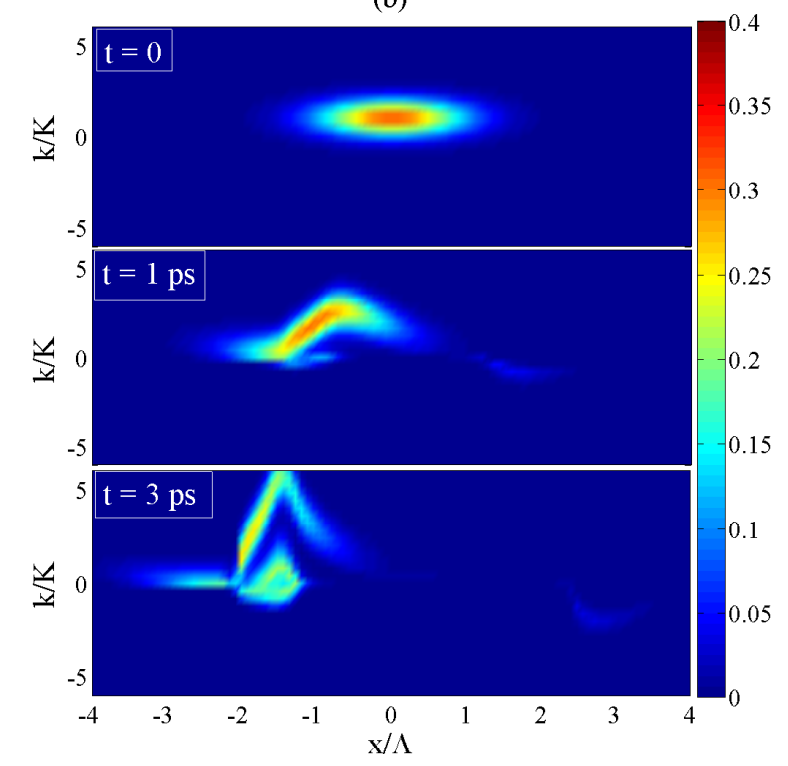

Figure 4: Hole density $\left(p^{+}(x, k)+p^{-}(x, k)\right)[p]$ at different snapshots for $N=250$ electrons/holes: (a) with scattering, (b) without scattering. Here $[p]=N / \Lambda K=1.25$.

density distributions, as shown in Fig. 5

\section{DIPOLE RADIATION: INTENSITY AND SPECTRUM}

In order to make relation of our results to possible experimental observations of the space-charge effects in the current evolution, here we study the radiation of a sin- 


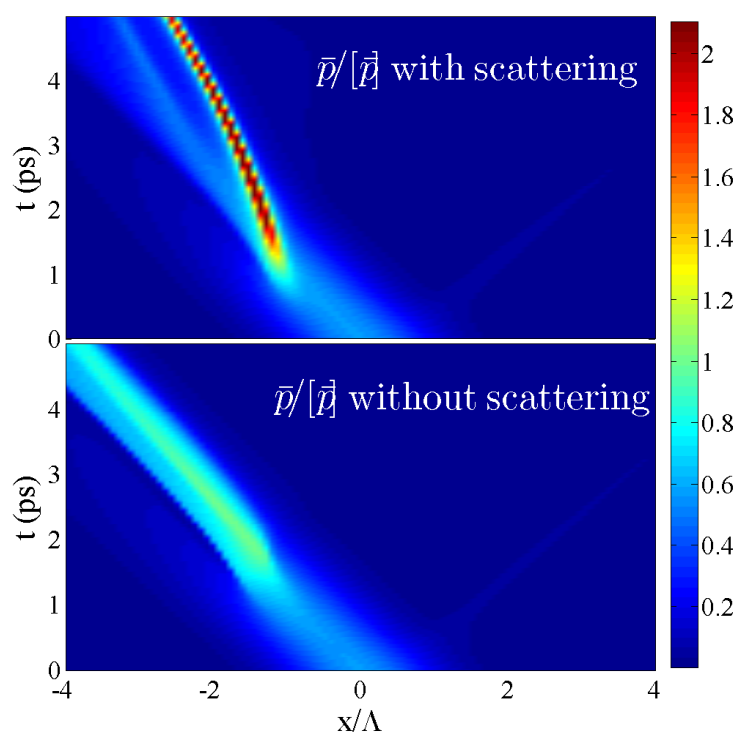

Figure $5: \bar{p}(x, t) /[\bar{p}]$ for $N=250$ electrons/holes. Here $[\bar{p}]=$ $N / \Lambda=2.5 \times 10^{6} \mathrm{~cm}^{-1}$.

gle nanotube after the current injection to see how the system parameters can be found from the radiation intensity and the spectrum. For this purpose we introduce the dipole moment as:

$$
D(t)=-e \int_{-\infty}^{+\infty}(\bar{p}(x, t)-\bar{n}(x, t)) x d x
$$

where the corresponding radiation intensity is proportional to $\left(d^{2} D / d t^{2}\right)^{2}$. The maximum value of $D$ can be estimated as $N|e| \Lambda$. Taking into account that the timescale of the process is $\Lambda / v_{0}$, we obtain the typical value of $d^{2} D / d t^{2}$ of the order of $|e| v_{0}^{2} \times N / \Lambda$. Taking into account the Pauli blocking limitations, $N / \Lambda \leq$ $K_{0}$, we obtain the fundamental limit for the derivative $d^{2} D / d t^{2} \leq e v_{0} \epsilon\left(k_{0}\right) / \hbar$. The corresponding spectral density for current injected at $t=0$ is given by

$$
I(\omega) \sim\left|\int_{0}^{+\infty} \frac{d^{2} D}{d t^{2}} e^{i \omega t} d t\right|^{2}
$$

Figure 6 shows $d^{2} D / d t^{2}$ for $N=250$ with and without scattering effects. The system parameters demonstrate themselves in the spectrum of the radiation. Increasing the number of carriers produces a sharp peak which is somewhat augmented and sharpened by scattering. Although the scattering sharpens the distributions, it only weakly modifies the integral parameter such as the dipole moment, as can be seen in Fig. 6. The Fourier transform of $d^{2} D / d t^{2}$ provides the spectrum of the radiation peaked at the frequency of $200 \mathrm{GHz}$ for a time window of $5 \mathrm{ps}$.

\section{CONCLUSIONS}

We have studied the time evolution of charge density after optical injection of a charge current in metallic carbon nanotubes with "relativistic" spectrum and identified different regimes of dynamics. The main impact on the carrier density evolution is produced by the spacecharge effects. However, due to the zero effective mass of the carriers, these Coulomb forces cannot prevent the calculated large separation of the electron and hole densities. This is in contrast to the relatively small separation expected in semiconductor structures, where electrons and holes have finite masses. Although the scattering of carriers by impurities and phonons considerably sharpens the density distribution, it does not influence strongly its integral characteristics such as the dipole moment resulting from the electron-hole separation, thereby rendering difficult the experimental verification of this effect. The time evolution of the dipole moment leads to a dipole radiation, which can be measured experimentally and provide information about the dynamics of the carriers. The spectral width of the radiation is mainly determined by the ratio of the "relativistic" velocity to the spatial width of the initial density distribution, while the intensity depends on the injected carrier density. Our results show that, as the intensity of the exciting radiation increases, there is a fundamental limit for the radiation intensity that is determined solely by the radiation frequency and related to Pauli blocking in the injection process. Realistic numerical parameters correspond to the radiation spectrum peaked at a fraction of a $\mathrm{THz}$, in the range of experimental observation of Ref. [6].

\section{ACKNOWLEDGEMENT}

This work was supported by the University of Basque Country UPV/EHU under program UFI 11/55, Spanish MICINN (projects FIS2011-28838-C02-01 and FIS201236673-C03-01), and "Grupos Consolidados UPV/EHU del Gobierno Vasco" (IT-472-10).

\section{APPENDIX}

Here we derive the expression for electric field and show the importance of the points where the derivative of the total charge density vanishes. Electric field is derived from the Coulomb forces for a singe-wall nanotube:

$$
E(x, t)=\int_{-\infty}^{\infty}[\bar{p}(x-s, t)-\bar{n}(x-s, t)] \mathcal{K}(s) d s
$$

with

$$
\mathcal{K}(s)=-\frac{2 e}{\pi \epsilon_{\perp}} \int_{0}^{\pi / 2} \frac{s d \theta}{\left(s^{2}+4 a^{2} \frac{\epsilon_{\|}}{\epsilon_{\perp}} \sin ^{2} \theta\right)^{3 / 2}}
$$


(a)

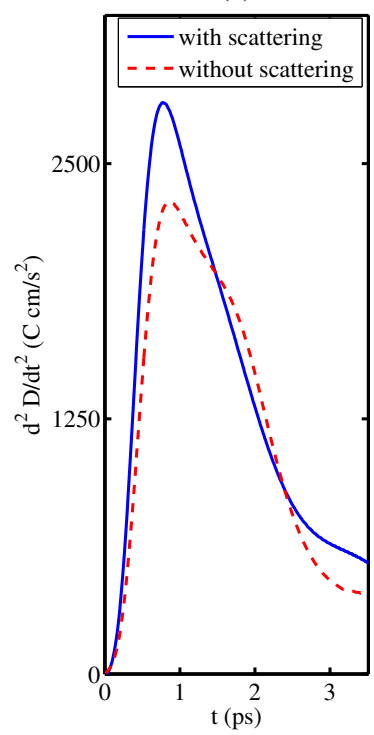

(b)

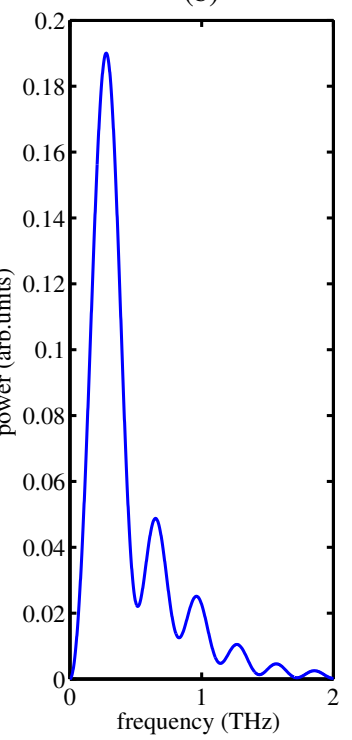

Figure 6: (a) Second time derivative of the dipole moment vs time and (b) power spectrum for $N=250$ electrons/holes. Scattering times are 2 ps for both types of carriers.

We describe ensemble of nanotubes of the radius $a$ as an anisotropic medium with the longitudinal and transversal permittivities $\epsilon_{\|}$and $\epsilon_{\perp}$, respectively $\underline{13117}$. The shape of $\mathcal{K}(s)$ in Eq. (A2) follows from the Poisson equation for

the electric potential of a point positive charge $-e$ at the origin,

$$
\left[\epsilon_{\|} \partial_{x}^{2}+\epsilon_{\perp} \nabla_{\perp}^{2}\right] \mathcal{V}=4 \pi e \delta(x) \delta\left(\mathbf{x}_{\perp}\right), \quad \mathbf{x}_{\perp}=(y, z),
$$

which can be written as

$$
\begin{gathered}
\left(\frac{\partial^{2}}{\partial \tilde{x}^{2}}+\frac{\partial^{2}}{\partial \tilde{y}^{2}}+\frac{\partial^{2}}{\partial \tilde{z}^{2}}\right) \mathcal{V}=\frac{4 \pi e}{\epsilon_{\perp \sqrt{\epsilon_{\|}}}} \delta(\tilde{x}) \delta(\tilde{y}) \delta(\tilde{z}), \\
\tilde{x}=\frac{x}{\sqrt{\epsilon_{\|}}},(\tilde{y}, \tilde{z})=\frac{1}{\sqrt{\epsilon_{\perp}}}(y, z) .
\end{gathered}
$$

In terms of the original variables, the solution is

$$
\begin{gathered}
\mathcal{V}=-\frac{e}{\epsilon_{\perp} \sqrt{x^{2}+\frac{\epsilon_{\|}}{\epsilon_{\perp}} \mathbf{x}_{\perp}^{2}}} \\
\frac{\partial \mathcal{V}}{\partial x}=\frac{e x}{\epsilon_{\perp}}\left[x^{2}+\frac{\epsilon_{\|}}{\epsilon_{\perp}} \mathbf{x}_{\perp}^{2}\right]^{-3 / 2} .
\end{gathered}
$$

The electric field (VII) is found straightforwardly by convolution of $-\partial \mathcal{V} / \partial x$ with the charge density $[\bar{p}(x, t)-$ $\bar{n}(x, t)] \delta\left(\left|\mathbf{x}_{\perp}\right|-a\right) / 2 \pi a$ and changing variables in the resulting integral. After integrating by parts, changing variables and using that the electron and hole densities rapidly decrease at large $|x| \gg \Lambda$, (A1) becomes

$$
\begin{gathered}
E(x, t)=\frac{e}{\pi \sqrt{\epsilon_{\|} \epsilon_{\perp}}} \frac{\partial}{\partial x} \int_{-\infty}^{+\infty} d s\left[\bar{p}\left(x-2 a s \sqrt{\frac{\epsilon_{\|}}{\epsilon_{\perp}}}, t\right)-\bar{n}\left(x-2 a s \sqrt{\frac{\epsilon_{\|}}{\epsilon_{\perp}}}, t\right)\right] \int_{0}^{\pi / 2} \frac{d \theta}{\left(s^{2}+\sin ^{2} \theta\right)^{1 / 2}} \\
=\frac{e}{\pi \sqrt{\epsilon_{\|} \epsilon_{\perp}}} \frac{\partial}{\partial x} \int_{-\infty}^{+\infty}\left[\bar{p}\left(x-2 a s \sqrt{\frac{\epsilon_{\|}}{\epsilon_{\perp}}}, t\right)-\bar{n}\left(x-2 a s \sqrt{\frac{\epsilon_{\|}}{\epsilon_{\perp}}}, t\right)\right] K\left(\frac{1}{\sqrt{s^{2}+1}}\right) \frac{d s}{\sqrt{s^{2}+1}} .
\end{gathered}
$$

Here we have written the integral over $\theta$ in terms of the complete elliptic integral of the first kind. Taking into account that the length scale of the electron and hole density distributions is of the order of $\Lambda \gg a$, one can approximate this expression as

$$
E(x, t) \sim \frac{e f}{\sqrt{\epsilon_{\|} \epsilon_{\perp}}} \frac{\partial}{\partial x}[\bar{p}(x, t)-\bar{n}(x, t)],
$$

with numerical factor

$$
f=\pi \ln 2+\frac{\pi^{2}}{8}+2 \int_{0}^{\infty}\left[K\left(\frac{1}{\sqrt{s^{2}+1}}\right)-\frac{\pi}{2}-\frac{\pi}{8 \sqrt{1+s^{2}}}\right] \frac{d s}{\sqrt{1+s^{2}}} \approx 5.43
$$

The approximate expression (A9) is a local relationship between the charge density and electric field, thereby 
depending on the geometric mean of the permittivities,

$\sqrt{\epsilon_{\|} \epsilon_{\perp}}$, to which both contribute equally.

1 A. Haché, Y. Kostoulas, R. Atanasov, J. L. P. Hughes, J. E. Sipe, and H. M. van Driel Phys. Rev. Lett. 78, 306 (1997).

2 Y. Kerachian, P. A. Marsden, H. M. van Driel, and A. L. Smirl, Phys. Rev. B 75, 125205 (2007).

3 M. J. Stevens, A. Najmaie, R. D. R. Bhat, J. E. Sipe, H. M. van Driel, and A. L. Smirl J. Appl. Phys. 94, 4999 (2003); H. Zhao, E. J. Loren1, A. L. Smirl and H. M. van Driel, J. Appl. Phys. 103, 053510 (2008).

4 A. Najmaie, R. D. R. Bhat, and J. E. Sipe, Phys. Rev. B 68, 165348 (2003).

${ }^{5}$ D. Sun, C. Divin, J. Rioux, J. E. Sipe, C. Berger, W. A. de Heer, P. N. First, and T. B. Norris, Nano Lett. 101293 (2010).

${ }^{6}$ R. W. Newson, A. A. Green, M. C. Hersam, and H. M. van Driel, Phys. Rev. B 83, 115421 (2011).

7 E. Ya. Sherman, R. M. Abrarov, and J. E. Sipe, Phys. Rev. B 80, 161308 (2009).

8 O. J. Korovyanko, C.-X. Sheng, Z. V. Vardeny, A. B. Dalton, and R. H. Baughman Phys. Rev. Lett. 92, 017403 (2004).
9 B. A. Ruzicka, R. Wang, J. Lohrman, S. Ren, and H. Zhao Phys. Rev. B 86, 205417 (2012).

10 F. Wang, D. J. Cho, B. Kessler, J. Deslippe, P. J. Schuck, S. G. Louie, A. Zettl, T. F. Heinz, and Y. R. Shen, Phys. Rev. Lett. 99, 227401 (2007).

11 Y. Chen, A. K. Ng, S. Bai, R.Si, L. Wei, and Q. Wang, Carbon Nanotubes and their Applications, edited by Q. Zhang (Pan Stanford Publishing, Singapore 2012), p. 128.

12 J. H. Grönqvist, M. Hirtschulz, A. Knorr, and M. Lindberg, Phys. Rev. B 81, 035414 (2010).

13 W. Lu, D. Wang, and L. Chen, Nano. Let. 7, 2729 (2007).

14 S. Piscanec, M. Lazzeri, F. Mauri and A. C. Ferrari, Eur. Phys. J. Special Topics 148, 159 (2007).

15 M. Lazzeri and F. Mauri, Phys. Rev. B 73, 165419 (2006).

${ }^{16}$ P. A. Sundqvist, F. J. Garcia-Vidal, and F. Flores, Phys. Rev. B 78, 205427 (2008).

17 T. O. Wehling, E. Sasoglu, C. Friedrich, A. I. Lichtenstein, M. I. Katsnelson, and S. Blügel, Phys. Rev. Lett. 106, 236805 (2011). 